\title{
Resting heart rate predicts all-cause mortality in sub-Saharan African patients with heart failure: a prospective analysis from the Douala Heart failure registry (Do-HF)
}

\begin{abstract}
Anastase Dzudie ${ }^{1,2,3}$, Blaise Barche ${ }^{2}$, Sidick Mouliom ${ }^{1}$, Ariane Nouko ${ }^{2}$, Raissa Fogue ${ }^{2}$, Jules Ndjebet ${ }^{4}$, Serah Abang Makoh ${ }^{5}$, Joseph Abah ${ }^{6}$, Armel Djomou ${ }^{7}$, Archange Nzali ${ }^{2}$, Clovis Nkoke ${ }^{2}$, Felicite Kamdem ${ }^{1}$, Samuel Kingue ${ }^{3,8}$, Karen Sliwa ${ }^{9}$, Andre Pascal Kengne ${ }^{10,11}$

${ }^{1}$ Cardiology and Cardiac Pacing Unit, Douala General Hospital, Douala, Cameroon; ${ }^{2}$ Clinical Research Education Networking \& Consultancy (CRENC), Douala, Cameroon; ${ }^{3}$ Faculty of Medicine and Biomedical Sciences, University of Yaounde I, Yaounde, Cameroon; ${ }^{4}$ Douala Cardiovascular Center, Douala, Cameroon; ${ }^{5}$ Mboppi Baptist Hospital, Douala, Cameroon; ${ }^{6}$ Douala Military Hospital, Cameroon; ${ }^{7}$ Clinique Cœur et vie, Douala, Cameroon; ${ }^{8}$ Yaounde General Hospital, Cameroon; ${ }^{9}$ Hatter Institute for Cardiovascular Research in Africa and the Institute of Infectious Disease and Molecular Medicine, Department of Medicine, Faculty of Health Sciences, University of Cape Town, Cape Town, South Africa; ${ }^{10}$ Department of Medicine, University of Cape Town, Cape Town, South Africa; ${ }^{11}$ Non-Communicable Diseases Research Unit, South African Medical Research Council, Cape Town, South Africa

Contributions: (I) Conception and design: A Dzudie, AP Kengne, B Barche; (II) Administrative support: A Dzudie; (III) Provision of study materials or patients: A Dzudie, S Molioum, F Kamdem, J Ndjebet, SA Makoh, J Abah, A Djomou, A Nzali; (IV) Collection and assembly of data: R Fogue, A Nouko; (V) Data analysis and interpretation: A Dzudie, AP Kengne, B Barche, R Fogue; (VI) Manuscript writing: All authors; (VII) Final approval of manuscript: All authors.

Correspondence to: Professor Anastase Dzudie, MD, PhD, FESC. Service of Internal Medicine and Cardiology, Douala General Hospital, 4856 Douala, Cameroon. Email: aitdzudie@yahoo.com.
\end{abstract}

Background: Higher resting heart rate (HR) is associated with mortality amongst Caucasians with heart failure (HF), but its significance has yet to be established in sub-Saharan Africans in whom HF differs in terms of characteristics and etiologies. We assessed the association of HR with all-cause mortality in patients with HF in sub-Saharan Africa.

Methods: The Douala HF registry (Do-HF) is an ongoing prospective data collection on patients with HF receiving care at four cardiac referral services in Douala, Cameroon. Patients included in this report were followed-up for 12 months from their index admission, for all-cause mortality. We used Cox-regression analysis to study the association of HR with all-cause mortality during follow-up.

Results: Of 347 patients included, 343 (98.8\%) completed follow-up. The mean age was 64 \pm 14 years, 176 $(50.7 \%)$ were female, and median admission HR was $85 \mathrm{bpm}$. During a median follow-up of 12 months, 78 (22.7\%) patients died. Mortality increased steadily with HR increase and ranged from $12.2 \%$ in the lower quartile of HR ( $\leq 69 \mathrm{bpm})$ to $34.1 \%$ in the upper quartile of HR (>100 bpm). Hazard ratio of 12 -month death per $10 \mathrm{bpm}$ higher HR was 1.16 (1.04-1.29), with consistent effects across most subgroups, but a higher effect in participants with hypertension vs. those without (interaction $\mathrm{P}=0.044$ ).

Conclusions: HR was independently associated with increased risk of all-cause mortality in this study, particularly among participants with hypertension. The implication of this finding for risk prediction or reduction should be actively investigated.

Keywords: Africa; heart rate (HR); heart failure (HF); mortality; outcome

Submitted Sep 21, 2020. Accepted for publication Jan 03, 2021.

doi: $10.21037 / \mathrm{cdt}-20-785$

View this article at: http://dx.doi.org/10.21037/cdt-20-785 


\section{Introduction}

High resting heart rate (HR) is as a strong predictor for adverse outcomes in several cardiovascular diseases including heart failure (HF) (1-3). In patients with suspected or proven coronary artery disease, resting HR was shown by Diaz et al. (4) to be a predictor of mortality. In HF Caucasians with reduced or preserved ejection fraction (HFrEF or HFpEF), with or without HF symptoms or signs, high HR has been correlated with adverse outcomes, independently of traditional risk factors (2-4). Accordingly, clinical trials on limiting HR medications used in HF like beta-blockers and ivabradine suggest that benefit comes from their effect in reducing $\operatorname{HR}(5,6)$. HR has therefore been considered as a target in $\mathrm{HF}$ and limiting HR medications are recommended to be used in HF by the European Society of Cardiology (ESC) (7). However, HR may differ by age and HF etiology and possibly other factors, such as ethnicity.

Given HF in sub-Saharan Africans occurs two decades earlier and is predominantly non-ischemic unlike western countries (8), it is uncertain whether HR would be a predictor of adverse outcomes in this specific population. Thus, we carried out this study to evaluate the prognostic value of HR in sub-Saharan African patients with HF. We hypothesized that higher HR would be associated with poor adverse outcomes.

We present the following article in accordance with the STROBE reporting checklist (available at: http://dx.doi. org/10.21037/cdt-20-785).

\section{Methods}

Written informed consent was obtained from each subject enrolled into the study. Patients were excluded if they refused to give informed consent. Ethical approval was obtained from the Cameroon National Ethics Committee before commencement of the registry by participating institutions (No. 2017/12/959/CE/CNERSH/SP), and the study conformed to the principles outlined in the Declaration of Helsinki (as revised in 2013).

\section{Study design and clinical setting}

The Douala HF registry (Do-HF) is a prospective, multisite, observational data collection on HF patients that was initiated in 2016 in four cardiology centers in the economic capital of Cameroon, central Africa. Douala is the largest city in Cameroon with a heterogeneous population. The centers were selected on the basis of availability of a clinical cardiologist able to perform echocardiography, and with previous experience in conducting cohort studies. Diagnosis of HF was in accordance with the ESC guidelines (7).

\section{Inclusion and exclusion criteria}

All patients aged 21 years and above with clinical signs and symptoms consistent with congestive HF (i.e., pedal oedema, elevated jugular venous pressure, pulmonary congestion, and tender hepatomegaly) and who were willing to continue follow-up for a minimum of 12 months were included.

\section{Data collection}

For all patients included in the registry, baseline resting HR was obtained from resting 12-lead ECG tracings undertaken as diagnostic workup. Other baseline characteristics included clinical signs and symptoms (NYHA classification), clinical parameters, transthoracic echocardiography parameters (ejection fraction and information on valvular dysfunction), laboratory investigations (serum electrolytes, renal function test), associated cardiovascular risk factors, and medications. Follow-up information was obtained from the registry with all-cause mortality being the outcome of interest.

\section{Statistical analyses}

SPSS $^{\circledR}$ version 25 (International Business Machines Corp., New York City) was used for analysis, HR was divided into quartiles based on distribution of baseline HR. Baseline characteristics overall and across HR quartiles are presented means and standard deviation for continuous variables and frequencies and percentages for categorical variables; with group comparisons via one-way analysis of variance and chi square test. Time-to-event analyses were based on cox proportional hazard regression models. The effect of baseline HR on mortality risk was tested in two ways: firstly, by computing the hazard ratio and $95 \%$ confidence interval for all-cause mortality across increasing quarters of baseline HR, using the lower quarter as reference; secondly, by fitting a continuous predictor to estimate the risk of death associated with a $10 \mathrm{bpm}$ increase in baseline HR, and consistency in the effects across major subgroups assessed 


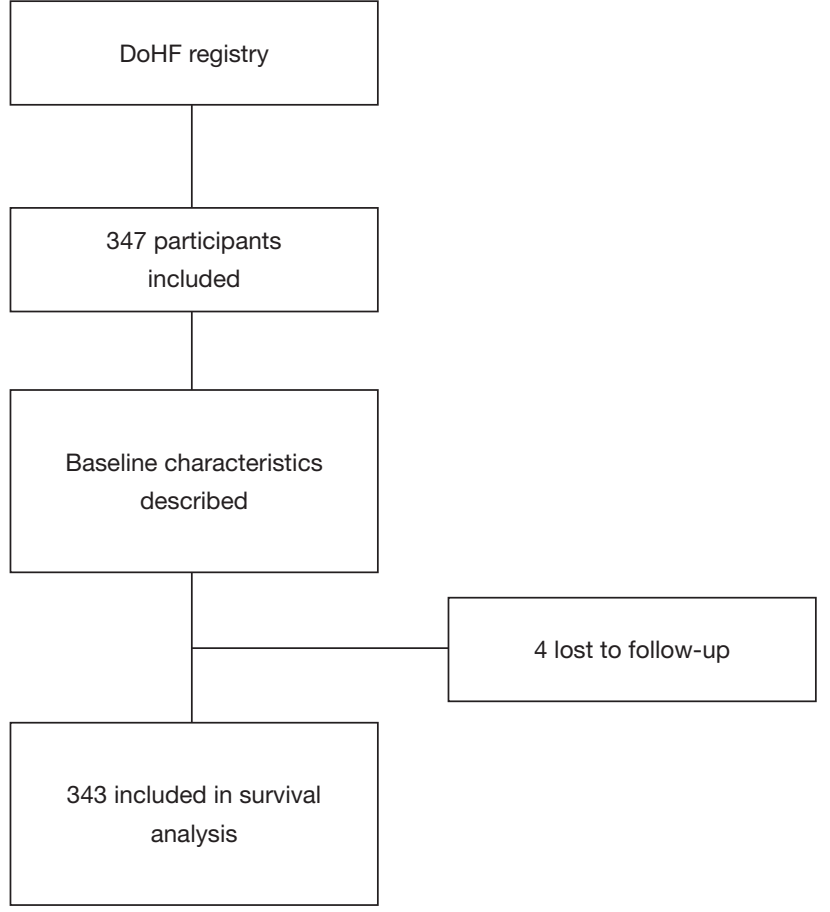

Figure 1 Flow diagram for participant recruitment. Do-HF, Douala Heart failure registry.

via interaction tests. Kaplan Meier estimator was use to illustrate the risk of death during follow-up across quarters of baseline HR, with comparison across quarters based on the logrank test. Statistical significance was considered for $\mathrm{P}<0.05$. Missing data as well as participants who were considered loss to follow up were excluded from Cox proportional regression model analysis

\section{Results}

\section{Patient characteristics}

Of the 347 patients included at baseline, 343 (98.8\%) had complete follow-up data (Figure 1). Baseline characteristics of these patients by HR quarters are presented in Table 1 . Overall, the mean age was $64 \pm 14$ years. Likewise, patients with the highest resting HR were younger and were more likely to have a history of HF in the past two years compared to those with lower resting HRs. Patients with higher resting HR were also likely to present with lower LVEF, lower estimated glomerular filtration rate when compared to the lower heart groups. There was no difference across the groups with regards to use of beta blockers, ACE inhibitors and angiotensin receptor blockers or loop diuretics.

\section{HR and survival}

During a mean follow-up of 12 months, 78 patients died. All-cause mortality at 1 year was significantly lower in the group with HR of $<69$ beats/min $(9 \%)$ when compared to patients with higher HR $(\mathrm{P}<0.001)$ (Figure 2). The pattern was similar in participant in sinus rhythm at baseline (logrank $\mathrm{P}=0.005)$ but only borderline in participants with atrial fibrillation or flutter at baseline (logrank $\mathrm{P}=0.089)$ (Figure 3).

Age-adjusted survival for the different HR groups is displayed in Figure 4, showing that those with higher HRs had shorter survival when compared to participants in the lower HR quarter. This pattern was observed in the overall sample (logrank $\mathrm{P}<0.001$ ), and within subgroups defined by the baseline presence or absence of atrial fibrillation/flutter, and presence or absence of sinus rhythm. However, due to small numbers, differences across HR quarters within subgroups were not always significant. Survival curves for participants with atrial fibrillation/flutter, and those in sinus rhythm at baseline are shown in Figure 3.

After adjusting for age, hypertension, diabetes mellitus, left ventricular ejection fraction, renal function cardiac rhythm, beta blocker use, and congestion; and using the lower quarter as reference in the overall sample, the hazard ratio of all-cause mortality was 2.45 (95\% CI: 0.93-6.44) in the second quarter, $1,70(0.63-4.65)$ in the third quarter and $2.98(1.13-7.89)$ in the top quarter. Small numbers precluded similar analyses in subgroups. With similar level of adjustment for confounders, the hazard ratios for every 10-beat increase in HR in the overall sample and across subgroups are represented in Figure 5. In the overall sample, each 10-beat higher HR was associated with a 16\% (95\% CI: 4-29\%) higher risk of 12-month mortality. In addition, congestion at baseline was associated with a $247 \%(95-518 \%)$ higher risk of mortality, while age, ejection fraction, hypertension, diabetes mellitus, use of betablockers and rhythm were not associated with risk of death during follow-up. Across complementary subgroups defined by gender (men $v s$. women), age ( $<60 v s . \geq 60$ years), setting of initial follow-up (inpatient $v s$. outpatients) renal function (altered $v s$. unaltered), rhythm (sinus vs. atrial flutter or fibrillation), ejection fraction (reduced, mid-range, preserved), use of betablocker (yes vs. no), congestion (yes $v s$. no), each 10 unit higher baseline HR was associated with similar increase in the risk of 12 -month mortality, with no evidence of statistical interaction (all interaction $\mathrm{P} \geq 0.31$ ); 
Table 1 Demographics, medical history, clinical and paraclinical findings, and medication use by heart rate group

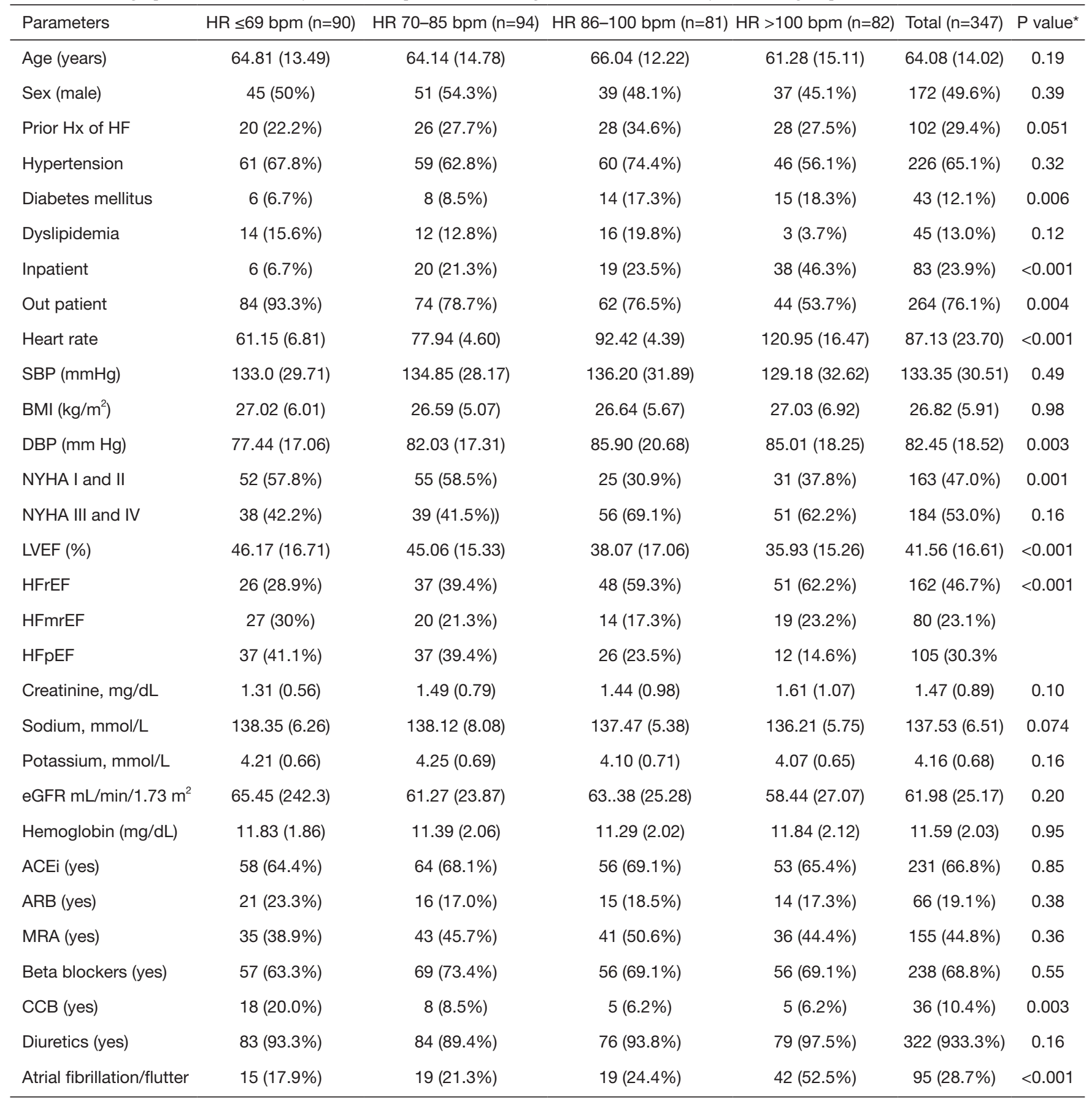

*, $\mathrm{P}$ value for linear trend. Presented are mean (standard deviation) for continuous variables and $\mathrm{n}(\%)$ for categorical variables. Statistics are computed from non-missing values, the number of which may vary from variable to variable. ACEi, angiotensin-converting enzyme inhibitors; ARB, angiotensin receptor blocker; CCB, calcium channel blockers; eGFR, estimated glomerular filtration rate; LVEF, left ventricular ejection fraction; HFmrEF, heart failure with moderately reduced ejection fraction; HFpEF, heart failure with preserved ejection fraction; HFrEF, heart failure with reduced ejection fraction; HR, hear rate; Hx of HF, history of heart failure; MRA, mineralocorticoid receptor antagonist 


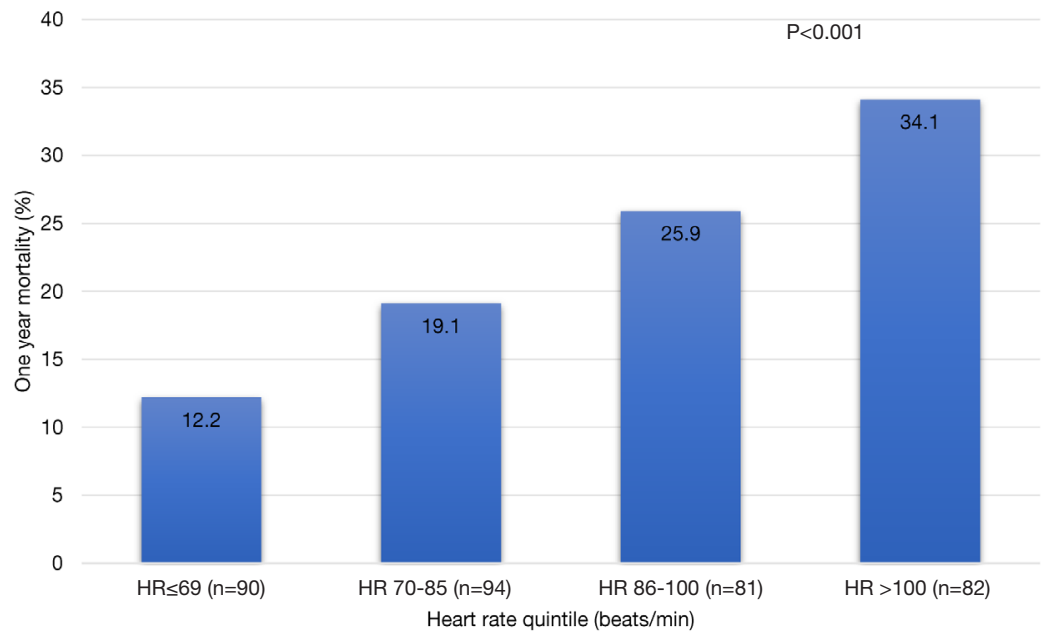

Figure 2 One-year all-cause mortality by baseline heart rate in patients with heart failure (the histogram).

Figure 5. There was however a significant interaction by status for hypertension on the effect of HR on all-cause mortality risk, with indication that the effect was more pronounced in participants with a history of hypertension [hazard ratio 1.30 (95\% CI: $1.16-1.45)$ ] than in those without [1.08 (0.96-1.23)], interaction $\mathrm{P}=0.044$.

\section{Discussion}

This study reveals that sub-Saharan African HF patients with higher resting HR have a significantly higher risk of mortality when compared to patients with lower resting HR, with each 10 -unit higher HR conferring a $16 \%$ higher risk of 12-month mortality. While being mostly consistent across most subgroups of participants, this effect appeared to be more important in participants with hypertension at baseline, compared to those without, resulting in significant statistical interaction. To our knowledge this is one of the rare studies on the predictive significance of HR rate for all-cause mortality in a cohort of HF patients in sub-Saharan Africa.

Our results are in agreement with those reported in several studies (9-12) most importantly these results are in line with two previous post-hoc analysis from the THESUS-HF study in which we evaluated the predictive value of several patient factors including ECG abnormalities on 6-month all-cause mortality in patients with HF $(13,14)$. Although the THESUS-HF did not focus on HR as a principal predictor of mortality, it was purely an African study (8), with patients of similar profile to those in the current study. Notably, the effect of increased HR was marked in the subgroup of patients with hypertension. Despite the variations of etiologies and clinical characteristics of HF globally, a similar effect has been reported in several studies (15-17). It shall be noted that hypertension has been reported as one of the common causes of HF in SSA (8). The enhanced effect of HR on mortality risk in the presence of hypertension can be explained by the elevated risk of arterial stiffness associated with increased HR and hypertension $(18,19)$, which in turn has been reported as a significant predictor of mortality (20).

Increased resting HR is a regular finding in HF. This results from a sustained vagal nerve activity inhibition and a hypersympathetic activity $(21,22)$. As HF progresses, there is an enhancement of the hypersympathetic activity representing an attempt to overcome the hemodynamic failure due to $\mathrm{HF}$ and to restore normal cardiac output; but this has been essentially studied in Caucasians (23-25). Regional differences in the patient characteristics, management, and outcomes of patients with HF have been described $(26,27)$. Also, differences in cardiovascular drug responses by different ethnic groups should be considered (28). The ESC recommends decreasing the HR in HF by adding ivabradine if beta-blockers do not attain the optimal HR (7). That only $68.8 \%$ of our patients received beta-blockers and median HR remains high is an indicator of nonoptimal therapy. Similarly these results strengthens the fact that despite variations in distribution of cardiovascular risk factors and etiologies of $\mathrm{HF}$ across the globe $(27,29)$, decreasing the HR to optimal values will as well improve outcome in HF patients in sub-Saharan Africa. 
A

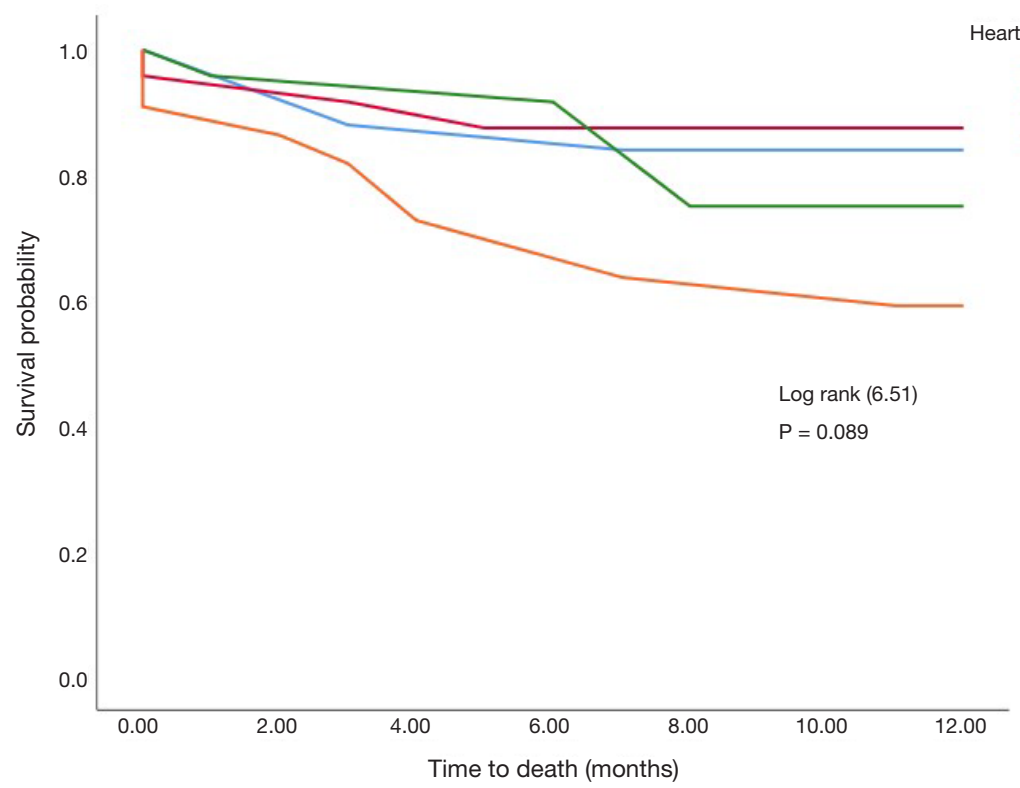

B

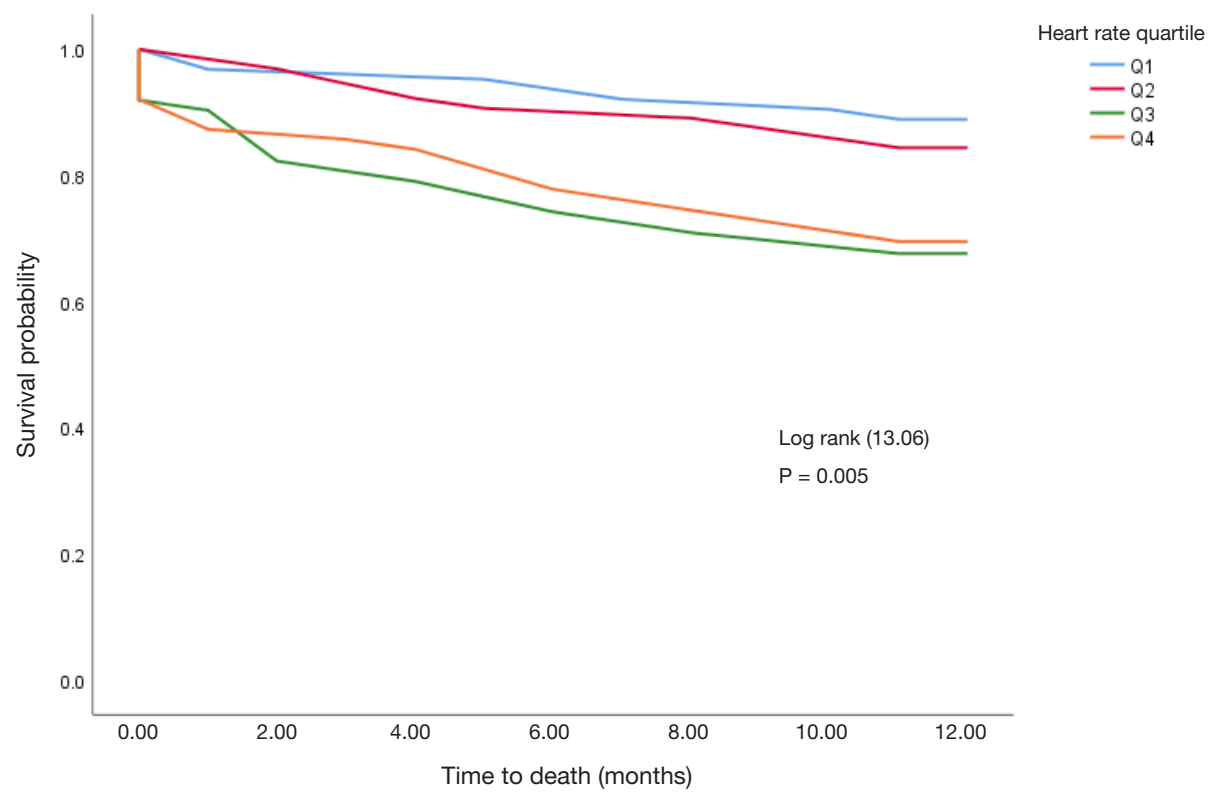

Figure 3 Survival curves of participants based on baseline sinus rhythm status. Survival curve in subgroup of patients (A) in atrial fibrillation/ flutter, (B) in sinus rhythm.

Our study has some weaknesses. First, we used single measurements of HR, assuming that they were representative of the usual HR of the patient. Actually, it is possible that resting HR might not be the best surrogate of HR during daily activities, a more accurate assessment would be through prolonged ambulatory ECG monitoring. However, Johansen et al. (30) described a good correlation between resting HR and mean rates on 24-hour monitoring in the general population as well as in ambulatory patients with HF (31). Secondly, our study group consisted of a general population seen in cardiology units, who may not necessarily represent the general population of patients with $\mathrm{HF}$ that are sometimes managed by internists and general practitioners. To our knowledge, this is the first report on the prognostic impact of HR in HF in sub-Saharan African black patients, a relatively young population when compared with Caucasians 


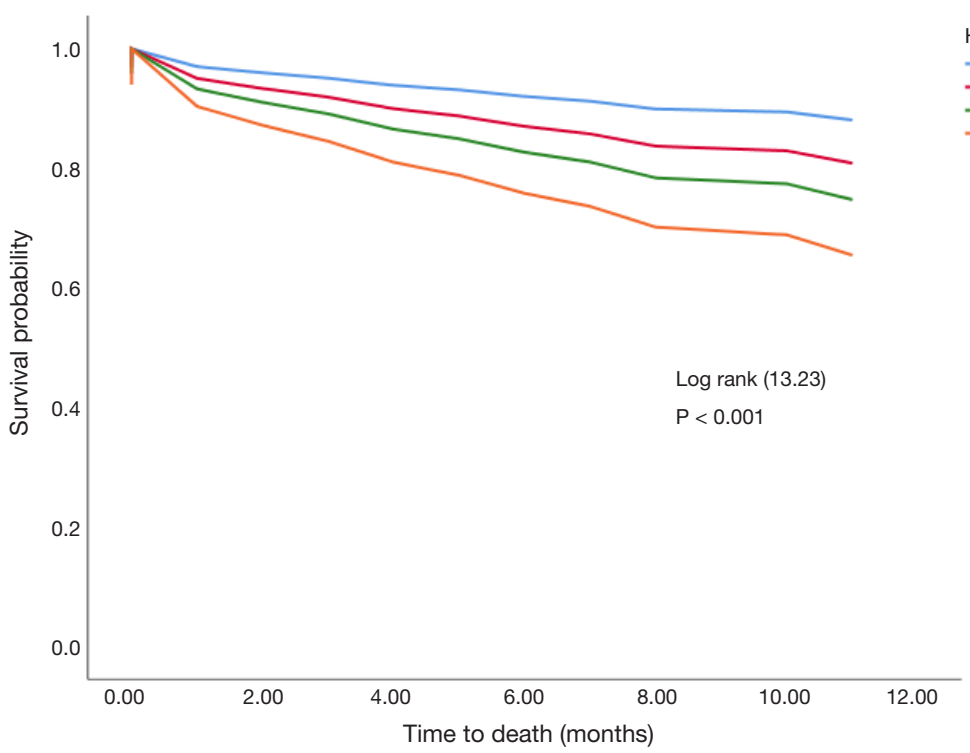

Figure 4 Kaplan-Meier survival curves showing statistically significant increase in all-cause mortality increases with increasing resting heart rate.

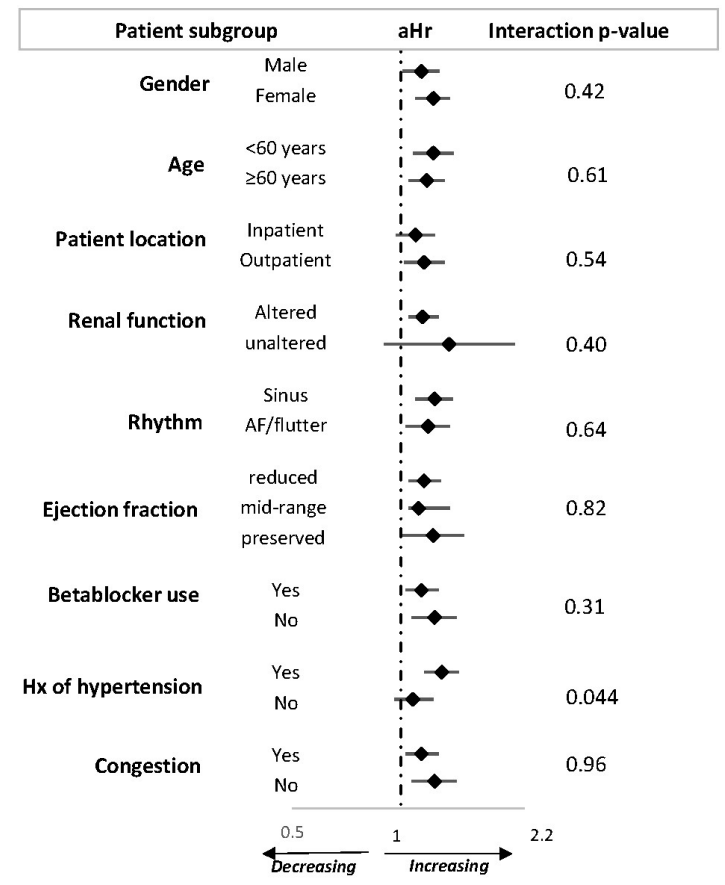

$\mathrm{AF}=$ atrial fibrillation, $\mathrm{aHr}=$ adjusted hazards ratio/ 10 beat increase in heart ratre

Figure 5 Adjusted hazard ratio and $95 \%$ confidence interval for each 10-unit higher heart rate in relation with all-cause mortality, across major subgroups of participants. AF, atrial fibrillation; $\mathrm{aHr}$, adjusted hazards ratio/10 beats increase in heart rate. and with predominantly non-ischemic etiology of HF. The fact that HR was obtained from resting 12-lead ECG tracings undertaken as diagnostic workup is a very important strength of our study. Indeed, in several other HF registries on the prognostic impact of $\mathrm{HR}$ in HF, previous exact timing of $\mathrm{HR}$ measurement was unclear.

\section{Conclusions}

In African patients with HF, resting HR is an independent risk factor for death, with a significantly higher effect in the subgroup of patients with hypertension, in line with reports from other regions around the world. As described by previous studies, the prescription of available therapies with proven efficacy in reducing deaths was rather suboptimal, especially for beta blockers. While additional studies are still warranted to determine if simple interventions designed to lower the HR will improve outcome in this population, optimization of HF therapy with prescription of beta blockers is urgent.

\section{Acknowledgments}

The authors wish to thank all the doctors, nurses and patients who participated in the registry. 
Funding: None.

\section{Footnote}

Reporting Checklist: The authors have completed the STROBE reporting checklist. Available at: http://dx.doi. org/10.21037/cdt-20-785

Data Sharing Statement: Available at: http://dx.doi. org/10.21037/cdt-20-785

Conflicts of Interest: All authors have completed the ICMJE uniform disclosure form (available at: http://dx.doi. org/10.21037/cdt-20-785). The authors have no conflicts of interest to declare.

Ethical Statement: The authors are accountable for all aspects of the work in ensuring that questions related to the accuracy or integrity of any part of the work are appropriately investigated and resolved. Written informed consent was obtained from each subject enrolled into the study. Patients were excluded if they refused to give informed consent. Ethical approval was obtained from the Cameroon National Ethics Committee before commencement of the registry by participating institutions (No. 2017/12/959/CE/CNERSH/SP), and the study conformed to the principles outlined in the Declaration of Helsinki (as revised in 2013).

Open Access Statement: This is an Open Access article distributed in accordance with the Creative Commons Attribution-NonCommercial-NoDerivs 4.0 International License (CC BY-NC-ND 4.0), which permits the noncommercial replication and distribution of the article with the strict proviso that no changes or edits are made and the original work is properly cited (including links to both the formal publication through the relevant DOI and the license). See: https://creativecommons.org/licenses/by-nc-nd/4.0/.

\section{References}

1. Hasenfuss G. Benefit of heart rate reduction in heart failure. Curr Heart Fail Rep 2010;7:156-8.

2. Ayesta A, Martínez-Sellés H, De Luna AB, et al. Prediction of sudden death in elderly patients with heart failure. J Geriatr Cardiol 2018;15:185-92.

3. Aune D, Sen A, ó'Hartaigh B, et al. Resting heart rate and the risk of cardiovascular disease, total cancer, and all-cause mortality - A systematic review and dose-response metaanalysis of prospective studies. Nutr Metab Cardiovasc Dis 2017;27:504-17.

4. Diaz A, Bourassa MG, Guertin MC, et al Long-term prognostic value of resting heart rate in patients with suspected or proven coronary artery disease. Eur Heart J 2005;26:967-74.

5. Tepper D. Effect of metoprolol CR/XL in chronic heart failure: Metoprolol CR/XL randomised intervention trial in congestive heart failure (MERIT-HF). Congest Heart Fail 1999;5:184-5.

6. The Cardiac Insufficiency Bisoprolol Study II (CIBIS-II): a randomised trial. Lancet 1999;353:9-13.

7. Ponikowski P, Voors AA, Anker SD, et al. 2016 ESC Guidelines for the diagnosis and treatment of acute and chronic heart failure: The Task Force for the diagnosis and treatment of acute and chronic heart failure of the European Society of Cardiology (ESC)Developed with the special contribution of the Heart Failure Association (HFA) of the ESC. Eur Heart J 2016;37:2129-200.

8. Damasceno A, Mayosi BM, Sani M, et al. The causes, treatment, and outcome of acute heart failure in 1006 Africans from 9 countries: Results of the subSaharan Africa survey of heart failure. Arch Intern Med 2012;172:1386-94.

9. Shang $X$, Lu R, Liu M, et al. Heart rate and outcomes in patients with heart failure with preserved ejection fraction: A dose-response meta-analysis. Medicine (Baltimore) 2017;96:e8431.

10. Swedberg K, Komajda M, Böhm M, et al. Ivabradine and outcomes in chronic heart failure (SHIFT): A randomised placebo-controlled study. Lancet 2010;376:875-85.

11. Zhang D, Shen X, Qi X. Resting heart rate and all-cause and cardiovascular mortality in the general population: A meta-analysis. CMAJ 2016;188:E53-63.

12. Castagno D, Skali H, Takeuchi M, et al. Association of heart rate and outcomes in a broad spectrum of patients with chronic heart failure: Results from the CHARM (Candesartan in Heart Failure: Assessment of Reduction in Mortality and morbidity) program. J Am Coll Cardiol 2012;59:1785-95.

13. Sliwa K, Davison BA, Mayosi BM, et al. Readmission and death after an acute heart failure event: Predictors and outcomes in sub-Saharan Africa: Results fromthe THESUS-HF registry. Eur Heart J 2013;34:3151-9.

14. Dzudie A, Milo O, Edwards C, et al. Prognostic significance of ECG abnormalities for mortality risk in acute heart failure: Insight from the Sub-Saharan Africa 
survey of heart failure (THESUS-HF). J Card Fail 2014;20:45-52.

15. Benetos A, Rudnichi A, Thomas F, et al. Influence of Heart Rate on Mortality in a French Population: Role of Age, Gender, and Blood Pressure. Hypertension 1999;33:44-52.

16. Jouven X, Diop BI, Narayanan K, et al. Cardiac Pacing in Sub-Saharan Africa: JACC International. J Am Coll Cardiol 2019;74:2652-60.

17. Ryu M, Bayasgalan G, Kimm H, et al. Association of resting heart rate and hypertension stages on all-cause and cardiovascular mortality among elderly Koreans: The Kangwha Cohort Study. J Geriatr Cardiol 2016;13:573-9.

18. Chen S, Li W, Jin C, et al. Resting heart rate trajectory pattern predicts arterial stiffness in a communitybased Chinese cohort. Arterioscler Thromb Vasc Biol 2017;37:359-64.

19. Li S, Chen W, Srinivasan SR, et al. Childhood Blood Pressure as a Predictor of Arterial Stiffness in Young Adults: The Bogalusa Heart Study. Hypertension 2004;43:541-6.

20. Vlachopoulos C, Aznaouridis K, Stefanadis C. Prediction of Cardiovascular Events and All-Cause Mortality With Arterial Stiffness. A Systematic Review and Meta-Analysis. J Am Coll Cardiol 2010;55:1318-27.

21. Eckberg DL, Drabinsky M, Braunwald E. Defective cardiac parasympathetic control in patients with heart disease. N Engl J Med 1971;285:877-83.

22. Hori M, Okamoto $H$. Heart rate as a target of treatment

Cite this article as: Dzudie A, Barche B, Mouliom S, Nouko A, Fogue R, Ndjebet J, Makoh SA, Abah J, Djomou A, Nzali A, Nkoke C, Kamdem F, Kingue S, Sliwa K, Kengne AP. Resting heart rate predicts all-cause mortality in sub-Saharan African patients with heart failure: a prospective analysis from the Douala Heart failure registry (Do-HF). Cardiovasc Diagn Ther 2021;11(1):111-119. doi: 10.21037/cdt-20-785 of chronic heart failure. J Cardiol 2012;60:86-90.

23. Floras JS. Sympathetic Nervous System Activation in Human Heart Failure. J Am Coll Cardiol 2009;54:375-85.

24. Kishi T. Heart failure as an autonomic nervous system dysfunction. J Cardiol 2012;59:117-22.

25. Packer M. The neurohormonal hypothesis: A theory to explain the mechanism of disease progression in heart failure. J Am Coll Cardiol 1992;20:248-54.

26. Riegel B, Moser DK, Rayens MK, et al. Ethnic Differences in Quality of Life in Persons With Heart Failure. J Card Fail 2008;14:41-7.

27. Dokainish H, Teo K, Zhu J, et al. Global mortality variations in patients with heart failure: results from the International Congestive Heart Failure (INTER$\mathrm{CHF}$ ) prospective cohort study. Lancet Glob Health 2017;5:e665-72.

28. Johnson JA. Ethnic Differences in Cardiovascular Drug Response. Circulation 2008;118:1383-93.

29. Chaturvedi N. Coronary disease ethnic differences in cardiovascular disease. Heart 2003;89:681-6.

30. Johansen CD, Olsen RH, Pedersen LR, et al. Resting, night-time, and $24 \mathrm{~h}$ heart rate as markers of cardiovascular risk in middle-aged and elderly men and women with no apparent heart disease. Eur Heart J 2013;34:1732-9.

31. Pastor-Pérez FJ, Manzano-Fernández S, Goya-Esteban R, et al. Heart rate control in chronic heart failure: Resting versus mean heart rate with prolonged ambulatory ECG recording. Int J Cardiol 2013;170:e45-7. 